\title{
Magnetic resonance in the antiferromagnetic and normal state of $\mathrm{NH}_{3} \mathrm{~K}_{3} \mathrm{C}_{60}$
}

\author{
Ferenc Simon ${ }^{1}$, András Jánossy $^{1, \dagger}$, Ferenc Murányi $^{1}$, Titusz Fehér $^{1}$, \\ Hideo Shimoda ${ }^{2, \ddagger}$, Yoshihiro Iwasa ${ }^{2}$, and László Forró ${ }^{3}$ \\ ${ }^{1}$ Technical University of Budapest, Institute of Physics, H-1521 \\ Budapest, PO BOX 91, Hungary \\ ${ }^{2}$ Japan Advanced Institute of Science and Technology, Tatsunokuchi, \\ Ishikawa 923-1292, Japan \\ ${ }^{3}$ Laboratoire de Physique des Solides Semicristallins, IGA Department de \\ Physique, Ecole Polytechnique Federal de Lausanne, 1015 Lausanne, Switzerland
}

(May 11, 2018)

\begin{abstract}
We report on the magnetic resonance of $\mathrm{NH}_{3} \mathrm{~K}_{3} \mathrm{C}_{60}$ powders in the frequency range of 9 to $225 \mathrm{GHz}$. The observation of an antiferromagnetic resonance below the phase transition at $40 \mathrm{~K}$ is evidence for an antiferromagnetically ordered ground state. In the normal state, above $40 \mathrm{~K}$, the temperature dependence of the spin-susceptibilty measured by ESR agrees with previous static measurements and is too weak to be explained by interacting localized spins in an insulator. The magnetic resonance line width has an unusual magnetic-field dependence which is large and temperature independent in the magnetically ordered state and decreases rapidly above the transition. These observations agree with the suggestion that $\mathrm{NH}_{3} \mathrm{~K}_{3} \mathrm{C}_{60}$ is a metal in the normal state and undergoes a Mott-Hubbard metal to insulator transition at $40 \mathrm{~K}$.

71.30.+h,74.70,76.50
\end{abstract}

The superconducting transition temperature, $T_{c}$, of cubic alkali metal $\mathrm{A}_{3} \mathrm{C}_{60}$ fullerides has a simple relation to the lattice constant [1]: $T_{c}$ increases with the ionic size of the alkali. This motivates the quest to synthesize large lattice parameter fullerides with $C_{60}$ in the $\left(\mathrm{C}_{60}\right)^{3-}$ charge state. The intercalation of ammonia into $\mathrm{Na}_{2} \mathrm{CsC}_{60}$ increased $T_{c}$ by nearly $20 \mathrm{~K}$, from $10.5 \mathrm{~K}$ to $29.7 \mathrm{~K}$ [2]. In contrast, superconductivity was not detected at ambient pressure in $\mathrm{NH}_{3} \mathrm{~K}_{3} \mathrm{C}_{60}$, the ammonia intercalated stable phase of $\mathrm{K}_{3} \mathrm{C}_{60}$ [3]. According to Raman scattering in $\mathrm{NH}_{3} \mathrm{~K}_{3} \mathrm{C}_{60}$ the charge state is $\left(\mathrm{C}_{60}\right)^{3-}$ and, at least along some directions, the nearestneighbor interball separation exceeds substantially that of $\mathrm{Rb}_{2} \mathrm{CsC}_{60}$, a superconductor with one of the highest transition temperatures [4]. The two materials differ in that $\mathrm{NH}_{3}$ intercalation expands the lattice without changing the cubic symmetry in $\mathrm{Na}_{2} \mathrm{CsC}_{60}$ while $\mathrm{NH}_{3} \mathrm{~K}_{3} \mathrm{C}_{60}$ has a face-centered orthorhombic structure. Zhou et al. questioned the importance of lower symmetry since superconductivity is recovered under hydrostatic pressure at a relatively high temperature $(28 \mathrm{~K}$ at $14.8 \mathrm{kbar}$ ) without any change in the crystallographic structure [4]. They suggested that the smaller transfer integrals at ambient pressures suppresses superconductivity and favors a Mott-Hubbard transition to an insulating ground state. The observation by Iwasa et al. [5] and by Allen et al. [6] of a phase transition with no structural change [7] at about $40 \mathrm{~K}$ and ambient pressure reinforces this view. The ESR intensity at $9 \mathrm{GHz}$ disappeared below $40 \mathrm{~K}$ but the static susceptibility, $\chi$, measured by SQUID, and the ${ }^{13} \mathrm{C}$ NMR [5] changed little. In spite of these somewhat contradicting results, Iwasa et al. [5] concluded that the ground state is an antiferromagnet.
Initially it was thought that the ordered magnetic moment is unusually small, $\sim 0.01 \mu_{B}$. Recently, Tou et al. [8] found that the ${ }^{13} \mathrm{C}$ line is significantly broadened below the transition, indicating an antiferromagnetically ordered ground state of $\mathrm{NH}_{3} \mathrm{~K}_{3} \mathrm{C}_{60}$ with an ordered moment of $1 \mu_{B} / \mathrm{C}_{60}$. In the interpretation of the ${ }^{13} \mathrm{C} \mathrm{NMR}$ [8] it was assumed that spins lie perpendicularly to the external field and the spin-flop(SF) field is small, less than $1 \mathrm{~T}$. The recent detection of damped oscillations in zero-field $\mu \mathrm{SR}$ [9] is a further signature of static magnetic moments below $40 \mathrm{~K}$. Just like the ground state, the normal state of $\mathrm{NH}_{3} \mathrm{~K}_{3} \mathrm{C}_{60}$ is also poorly understood. In particular, it is unknown whether above $40 \mathrm{~K}$ it is a metallic or insulating. Initially, the temperature variation of $\chi$ and ${ }^{13} \mathrm{C}$ NMR $T_{1}$ of [5] were interpreted assuming a narrow band metallic normal state. Recent NMR data 8] were analyzed assuming localized spins on the $\mathrm{C}_{60}$ ions above $170 \mathrm{~K}$. A further complication arises from the recently found structural transition [7] due to the ordering of the $\mathrm{K}-\mathrm{NH}_{3}$ pairs at $T_{S}=150 \mathrm{~K}$.

The motivation for the present work is two-fold: following Ref. [10] on $\mathrm{RbC}_{60}$ and $\mathrm{CsC}_{60}$ we observe the antiferromagnetic resonance(AFMR) in $\mathrm{NH}_{3} \mathrm{~K}_{3} \mathrm{C}_{60}$ using a multi-frequency spin resonance technique. Our less detailed earlier work 11] was inconclusive in this respect. Below $40 \mathrm{~K}$ the $9 \mathrm{GHz}$ resonance spectrum of $\mathrm{NH}_{3} \mathrm{~K}_{3} \mathrm{C}_{60}$ is broad and good sensitivity and high purity material is required for its detection [11]. The AFMR in powders becomes narrower at higher fields and is an unambiguous evidence for the antiferromagnetic $(\mathrm{AF})$ ground state. Secondly, we gain information about the normal state from the ESR measured in a large frequency range. Our normal state ESR susceptibility data agree with the 
static susceptibility data of Ref. [5] and point to a metallic system rather than an insulator with interacting localized moments.

Several $\mathrm{NH}_{3} \mathrm{~K}_{3} \mathrm{C}_{60}$ powder samples were prepared following methods described elsewhere [5]. Samples were sealed in quartz ESR tubes under low pressure He. Powder X-ray diffraction showed them to be of high purity. The small residual $\mathrm{K}_{3} \mathrm{C}_{60}$ content was unobservable by $\mathrm{X}$-ray diffraction but increased the noise of the $9 \mathrm{GHz}$ ESR spectrometer below $19 \mathrm{~K}$. The main features of the ESR spectra were identical for all samples studied and we quote in our figures results from two samples. $9 \mathrm{GHz}$ ESR spectra were recorded on a commercial Bruker spectrometer. The 35, 75, 150 and $225 \mathrm{GHz}$ ESR were studied at the Budapest high frequency spectrometer. Samples were slowly $(\sim 50 \mathrm{~K} / \mathrm{h})$ cooled from $300 \mathrm{~K}$ to $5 \mathrm{~K}$. At $9 \mathrm{GHz}$ only data between 20 and $300 \mathrm{~K}$ are shown, as below $19 \mathrm{~K}$ residual $\mathrm{K}_{3} \mathrm{C}_{60}$ distorted the spectra. ESR intensities and g-factors were measured with respect to standard calibrating samples.

In Fig. 1 we show $\chi$ determined from the ESR intensity together with $\chi$ measured in Ref. [5] by SQUID technique. $\chi$ of $\mathrm{NH}_{3} \mathrm{~K}_{3} \mathrm{C}_{60}$ varies little through the 40 $\mathrm{K}$ transition for all ESR frequencies, $f$, between 9 (0.3 $\mathrm{T})$ and $225 \mathrm{GHz}(8.1 \mathrm{~T})$. Our $\chi$ data are in remarkable agreement with that of the SQUID results of Iwasa et al. [5] in the full temperature range. The SQUID measurements were corrected for the core electron contribution. ESR measures the spin susceptibility in the paramagnetic state and, if the applied field is large compared to the SF field, in the AF state also. Only the perpendicular AF susceptibility is observed in such large fields. The (molar) susceptibility below $T_{N}$ is $\chi_{\perp}=N_{A} \frac{\mu_{\text {eff }}^{2}}{3 k_{B}} \frac{1}{2 T_{N}}$, where $N_{A}$ is the Avogrado number. From Fig. $1 \chi_{\perp} \simeq 1.3 * 10^{-3}$ $\mathrm{emu} / \mathrm{mol}$ and the ordered moment is $\mu_{\mathrm{eff}}=0.9 \mu_{B} / \mathrm{C}_{60}$.

The variation of $\chi(T)$ with $T$ is difficult to explain with a "textbook" metallic or localized spin susceptibility. The variation is too weak to originate from a CurieWeiss temperature dependence of localized spins. The $3 \mathrm{D} \mathrm{AF}$ order sets in at a Neél temperature of $T_{N} \simeq 40$ K. Between 40 and $300 \mathrm{~K} \chi(T)$ decreases by a factor $\sim 2.3$ whereas a factor 4.25 would be expected from a Curie-Weiss behavior of antiferromagnetically correlated spins $\chi(T)=\frac{C}{T+T_{N}}$, where $\mathrm{C}$ is the Curie constant. The fit of $\chi(T)$ to a Curie-Weiss behavior with $T_{N}$ left as a free parameter leads to $T_{N} \geqq 120 \mathrm{~K}$ in contradiction with the observed long range $3 \mathrm{D}$ order at $40 \mathrm{~K}$. On the other hand, the variation of $\chi$ is larger than usually found in metallic systems. Nevertheless, strong correlation effects in a narrow band metal may lead to the observed variation of $\chi(T)$ as it was originally suggested in Ref. [5]. Electron-electron correlation effects are needed to explain the large value of $\chi$ of the $\mathrm{K}_{3} \mathrm{C}_{60}$ parent compound [1] also. The larger lattice constants of $\mathrm{NH}_{3} \mathrm{~K}_{3} \mathrm{C}_{60}$ suggest a narrower band and even more important correlation ef- fects. There is a slight anomaly in $\chi(T)$ at the structural transition, $T_{S}$. The transition is of second order and affects $\chi$ in the range of $50-150 \mathrm{~K}$. The sensitivity of $\chi(T)$ to the structural transformation is readily understood if the material is a metal with strong electron correlations since the freezing of molecular rotation is certainly accompanied by a change in electronic structure. In summary, above $T_{N}$ the material is more likely a strongly correlated metal than an insulator with localized spins. Unfortunately, a definite answer is not possible from the $\chi$ data alone.

Fig. 2 shows the variation of the resonance line width $(\Delta H)$ with temperature at various frequencies. The $40 \mathrm{~K}$ transition is marked by a sharp increase of $\Delta H$ with decreasing $T$. The 9,35 , and $75 \mathrm{GHz}$ spectra below $40 \mathrm{~K}$ are characteristic of an AFMR of a powder in applied fields larger than the SF field [10]: the line width narrows and the resonance field shifts proportionally to the inverse of the applied field. This is in contrast with paramagnetic systems where (excluding crystal field effects) $\Delta H$ is either independent of $f$ or increases with $f$. We rule out a spin-glass ground state, proposed in Ref. 12 for $\mathrm{RbC}_{60}$, since we observed no thermal or magnetic history dependence. Also, were the ground state a spin-glass, there would be no simple explanation for the scaling of the line shape. As shown in Fig. 3, the $24 \mathrm{~K}$ line shapes are identical at 9 and $35 \mathrm{GHz}$ if the magnetic field axis is multiplied by the resonance field, $H_{0}$. The close similarity of the scaled line shapes is a characteristic feature of AFMR in powders and implies a $1 / H_{0}(1 / f)$ dependence of $\Delta H$ below $40 \mathrm{~K}$. As expected, below $T=40 \mathrm{~K}$ the resonance field shift (not shown), $H_{0}(T)-H_{0}(40 \mathrm{~K})$ scales also with $1 / H_{0}$ at 9 and $35 \mathrm{GHz}$.

Between 75 and $225 \mathrm{GHz}$ the line width depends little on $f$ and does not follow the $1 / H_{0}$ field dependence (Fig. 4.). To explain this, we assume that in this range the $1 / f$ narrowing of the AFMR line is compensated by a g-factor anisotropy broadening linear in $f$. The g-factor anisotropy is a consequence of the orthorhombic crystal structure of $\mathrm{NH}_{3} \mathrm{~K}_{3} \mathrm{C}_{60}$ [3]. For simplicity, we assume in the following a uniaxial anisotropy and that the principal axes of the g-tensor coincide with the easy and hard axes of the AF state. In a single crystal the resonance field depends on the alignment of the external field with respect to the magnetic axes of the antiferromagnet. The hard and easy axis modes correspond to external fields perpendicular and parallel to the easy axis, respectively [13. If $H_{0}$ is larger than the SF field, $H_{S F}$, the modes are 13. $\omega_{ \pm}=\gamma_{ \pm} \sqrt{H_{0}^{2} \pm H_{S F}^{2}}$. The $+(-)$ sign corresponds to the hard (easy) axis mode, $\gamma_{ \pm}=\gamma \pm \Delta \gamma / 2$ where $\gamma=g \mu_{B} / \hbar, \Delta \gamma$ measures the anisotropy. In a powder of independent single crystals the spectrum has extrema at $H_{A F M R}^{ \pm}=\sqrt{\omega^{2} / \gamma_{ \pm}^{2} \mp H_{S F}^{2}},(\omega=2 \pi f)$. We assume that the measured linewidth $\Delta H=H_{A F M R}^{-}-H_{A F M R}^{+}$. A fit to this formula (Fig. 4) with the free parameters 
$H_{S F}$ and $\Delta \gamma$ is in good agreement with experiment. We find $\Delta \gamma=1300 \pm 200 \mathrm{ppm}$ independent of $T$ below $40 \mathrm{~K}$ and $H_{S F}(5 \mathrm{~K})=0.17 \mathrm{~T}$. The anisotropy in $\mathrm{NH}_{3} \mathrm{~K}_{3} \mathrm{C}_{60}$ is large in comparison with $\Delta \gamma / \gamma \simeq 80 \mathrm{ppm}$ in the orthorhombic fulleride polymer $R b \mathrm{C}_{60}$ at $T_{N}$. On the other hand, $H_{S F}$ in $\mathrm{NH}_{3} \mathrm{~K}_{3} \mathrm{C}_{60}$ is close to values in the $R b \mathrm{C}_{60}$ and $C s \mathrm{C}_{60}$ fulleride antiferromagnets [10] The $\mathrm{SF}$ field is $H_{S F}=\left(2 H_{E} H_{A}\right)^{1 / 2}$ where $H_{E}$ and $H_{A}$ are the exchange and anisotropy fields, respectively. $\mathrm{NH}_{3} \mathrm{~K}_{3} \mathrm{C}_{60}$ is composed of light elements where spin-orbit interactions are small and $H_{A}$ arises from dipolar fields and is of the order of $1 \mathrm{mT}$. Since $g \mu_{B} H_{E}$ is of the order of $k T_{N}$, we expect $H_{S F}$ is of the order of 0.1-1 T.

The assignment of the resonance below $40 \mathrm{~K}$ to an AFMR powder spectrum with a g-factor anisotropy implies that the broadening is inhomogeneous. However, although the spectra are significantly broadened, the line shapes are Lorentzian, which is characteristic of a homogeneous relaxational broadening. We do not know of any relaxational mechanism that could explain the data. It may be that the interaction at the boundaries of small domains with differing crystal orientations reduces the inhomogeneous broadening of the AFMR and renders the lineshape approximately Lorentzian. In this case the values of $H_{S F}$ and $\Delta \gamma / \gamma$ are only lower limits.

The ESR in the normal state (above $40 \mathrm{~K}$ ) has an interesting frequency and temperature dependence (Fig. 2, inset and Fig. 5). The line width, $\Delta H$, can be decomposed into $\Delta H(f, T)=\Delta H_{0}(T)+\Delta H_{f}(T)$, where $\Delta H_{f}(T)$ is proportional to $f$. The frequency independent term $\Delta H_{0}(T)$ has a maximum at the structural transition, $T_{S}$. This maximum is observed in the $9 \mathrm{GHz}$ line width but is hidden at higher frequencies by the frequency dependent term (Fig. 2, inset). As the transition is approached from above, molecular rotations of the $\mathrm{NH}_{3}$ groups become slower and fluctuations at the Larmor frequency broaden the line. Below the transition the molecular order increases gradually and the line narrows. A static or slowly fluctuating disorder is the usual mechanism for conduction electron spin relaxation and a maximum is expected if the state is metallic.

An incompletely resolved g-factor anisotropy is the most likely origin of $\Delta H_{f}(T)$ in orthorhombic $\mathrm{NH}_{3} \mathrm{~K}_{3} \mathrm{C}_{60}$. ( $\Delta H$ is independent of frequency in cubic $\left.\mathrm{K}_{3} \mathrm{C}_{60}\right) . \Delta H_{f}(T)=H_{0} \Delta \gamma / \gamma$ is proportional to $f$ up to $225 \mathrm{GHz}$ at and above $40 \mathrm{~K}$. As shown in Fig. 5, $\Delta \gamma / \gamma$ increases strongly as the temperature approaches $T_{N}$ from above. The g-factor anisotropy at $40 \mathrm{~K}$, (i.e. slightly above $T_{N}$ ) is equal to the temperature independent value in the $\mathrm{AF}$ state. The strong non-linearity in the frequency dependence of the linewidth appears suddenly at temperatures slightly below $40 \mathrm{~K}$. Thus fields up to $8 \mathrm{~T}$ do not smear the transition. Such behavior may be expected for an $\mathrm{AF}$ ordering with a large value of $H_{E}$ but is unlikely in a spin glass where the melting temperature is smeared by magnetic fields. The g-factor broadening must be incomplete in the paramagnetic state for the same reason as in the AF state. Although at $45 \mathrm{~K}$ the ESR is several times broader at $225 \mathrm{GHz}$ than at $9 \mathrm{GHz}$ the line shape is Lorentzian in both cases. The ESR of high purity $\mathrm{Al}$ is an example for an incomplete g-factor anisotropic broadening [14 in a metal. In $\mathrm{NH}_{3} \mathrm{~K}_{3} \mathrm{C}_{60}$, the increase of the g-factor anisotropy may be due to a gradual metal to insulator transition where the motional narrowing due to the diffusion of conduction electrons becomes less effective as $T_{N}$ is approached.

The observation of an AFMR below $40 \mathrm{~K}$ is an unambiguous evidence for an antiferromagnetically ordered ground state. The static susceptibility and ESR are compatible with a paramagnetic metallic state at high temperature. This could happen if $\mathrm{NH}_{3} \mathrm{~K}_{3} \mathrm{C}_{60}$ undergoes a Mott-Hubbard metal to insulator transition. If this suggestion is true then $\mathrm{NH}_{3} \mathrm{~K}_{3} \mathrm{C}_{60}$ is one of the rare examples, and would be the only example among fulleride compounds, in which a Mott-Hubbard transition takes place at experimentally accessible temperatures.

Support from the JSPS-HAS Japanese-Hungarian Cooperation Program, the Hungarian State Grants OTKA T029150, FKFP0352-1997, and HAS-TUB No. 04119 on Solids in Magnetic Fields, JSPS (RFTF96P00104), the Ministry of Education, Science, Sports, and Culture of Japan, and the Swiss NSF are acknowledged.

$\dagger \quad$ Corresponding author: atj@power.szfki.kfki.hu

¥ Present address: Department of Physics and Astronomy, University of North Carolina at Chapel Hill, Chapel Hill, NC27599, USA

[1] For a review see: O. Gunnarsson, Rev. Mod Phys. 69, 575 (1997).

[2] O. Zhou et al., Nature (London) 362, 433 (1993).

[3] M. J. Rosseinsky et al., Nature (London) 364, 425 (1993).

[4] O. Zhou et al., Phys. Rev. B 52, 483 (1995).

[5] Y. Iwasa et al., Phys. Rev. B 53, 8836 (1996).

[6] K. M. Allen et al., J. Mater. Chem. 6, 1445 (1996).

[7] K. Ishii et al., Phys. Rev. B 59, 3956 (1999).

[8] H. Tou et al., Phys. B 261, 868 (1999).

[9] K. Prassides et al., Phys. C 282, 307 (1997); K. Prassides et al., J. Am. Chem. Soc. to be published.

[10] A. Jánossy et al., Phys. Rev. Lett. 79, 2718 (1997).

[11] F. Simon et al., Proceedings of IWNEPNM98. World Scientific, 1998, pp296. Singapore.

[12] M. Bennati et al., Phys. Rev. B 58, 15603 (1998).

[13] S. Foner, Antiferromagnetic and Ferrimagnetic Resonance in Magnetism, edited by G. T. Rado and H. Suhl (Academic, New York, 1963), Vol. I, p. 384.

[14] D. Lubzens et al., Phys. Rev. Lett. 29, 1387 (1972); D. R. Fredkin et al., Phys. Rev. Lett. 29, 1390 (1972). 
Figure Captions:

Fig. 1. Spin-susceptibility of $\mathrm{NH}_{3} \mathrm{~K}_{3} \mathrm{C}_{60}$ measured by ESR at $9 \mathrm{GHz}(\boldsymbol{\square} 0.3 \mathrm{~T})$ and $225 \mathrm{GHz}(\diamond 8.1 \mathrm{~T})$. Solid curve is SQUID data from Ref. [5] measured at $1 \mathrm{~T}$ after core electron diamagnetic susceptibility correction. $T_{S}$ is the structural, $T_{N}$ the magnetic ordering temperature.

Fig. 2. Temperature dependence of the line width, $\Delta H$, at various frequencies. $(\boldsymbol{\square} \mathrm{GHz}, \bigcirc 35 \mathrm{GHz}, \boldsymbol{\Delta} 75$ $\mathrm{GHz}$ ) Inset shows $\Delta H$ (in $\mathrm{mT}$ ) above $40 \mathrm{~K}$.

Fig. 3. Antiferromagnetic resonance at $24 \mathrm{~K}$. Line shapes at 9 and $35 \mathrm{GHz}$ are identical if the magnetic field scale is multiplied by the resonance field, $H_{0}$. (Low intensity narrow impurity lines are subtracted from the spectra.) We chose the normalizing constant $K=0.17$ $T$, the spin flop field $H_{S F}$ measured at $5 \mathrm{~K}$.

Fig. 4. $\Delta H$ as a function of $f$ at various temperatures. The narrowing of the AFMR is compensated at high frequencies by a g-factor anisotropy broadening. (Solid lines: fit, explained in text.) Inset: $\Delta H$ (in $\mathrm{mT}$ ) is linear in $f$ due to partially resolved g-factor anisotropy. (Dashed lines: linear fit.)

Fig. 5. Temperature variation of normalized linearly $f$ dependent contribution. (Dashed line indicates $T_{N}$.) $\Delta \gamma / \gamma$ is assumed to be due to partially resolved g-factor anisotropy in both the AF and paramagnetic state. 


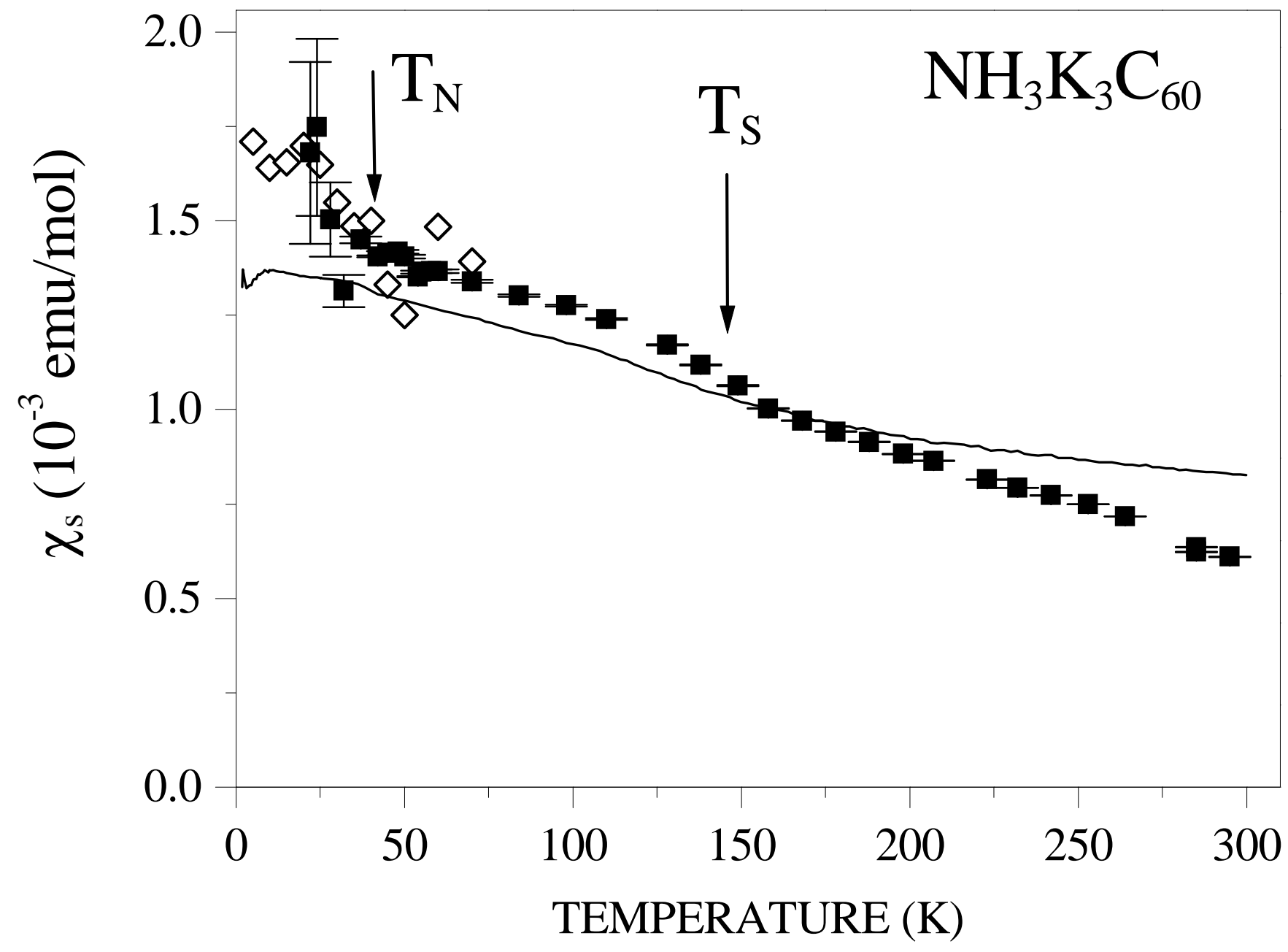




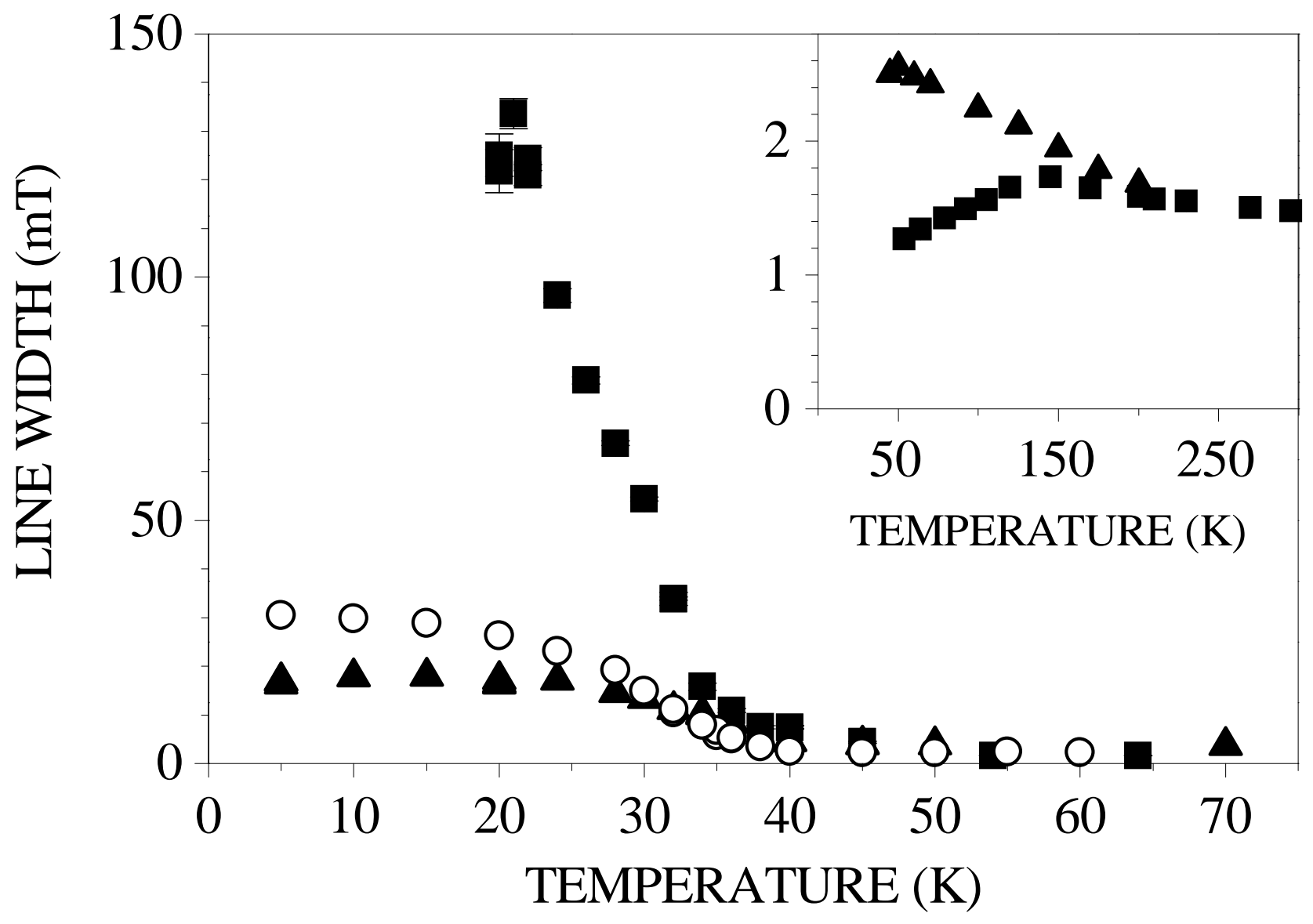




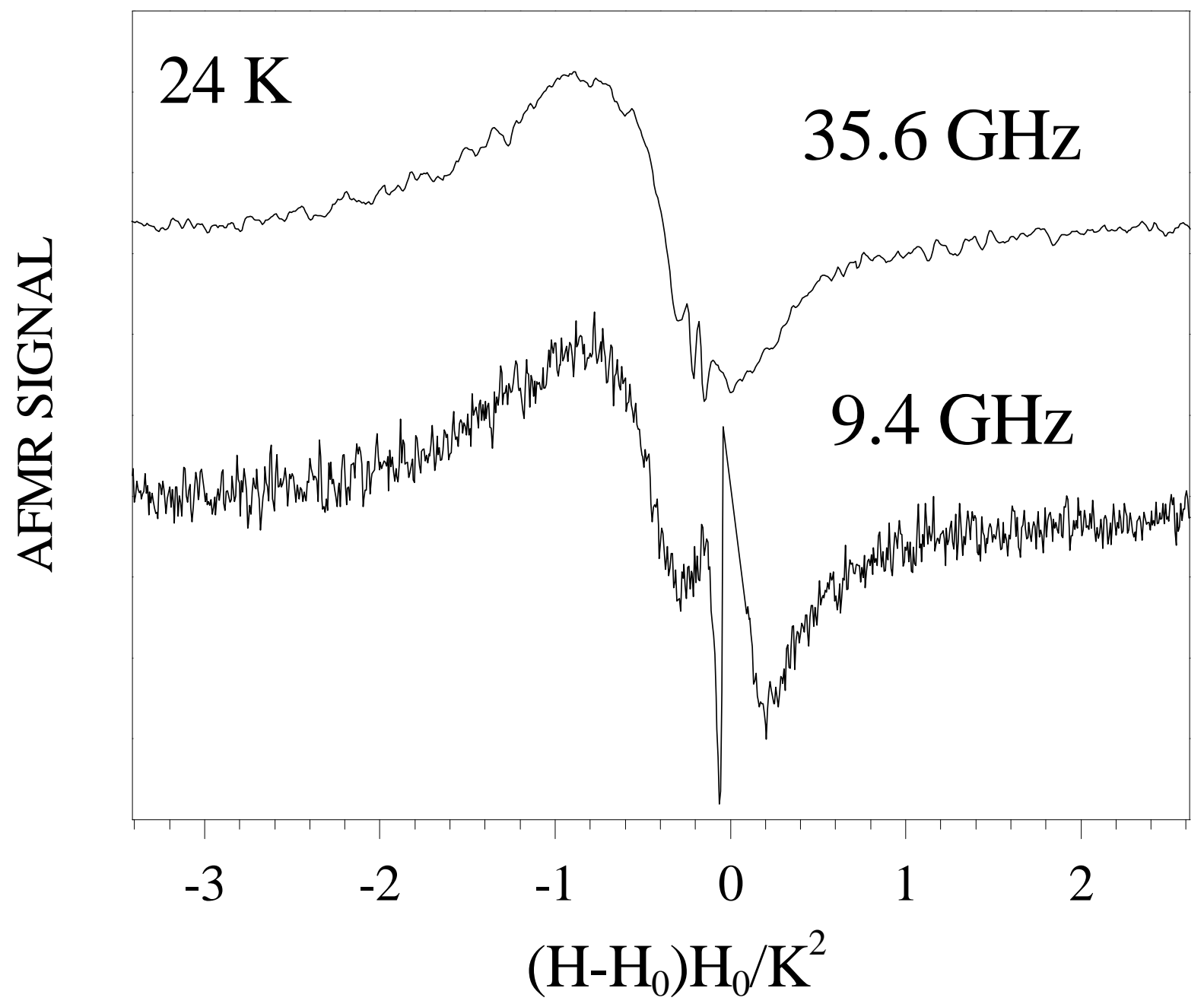




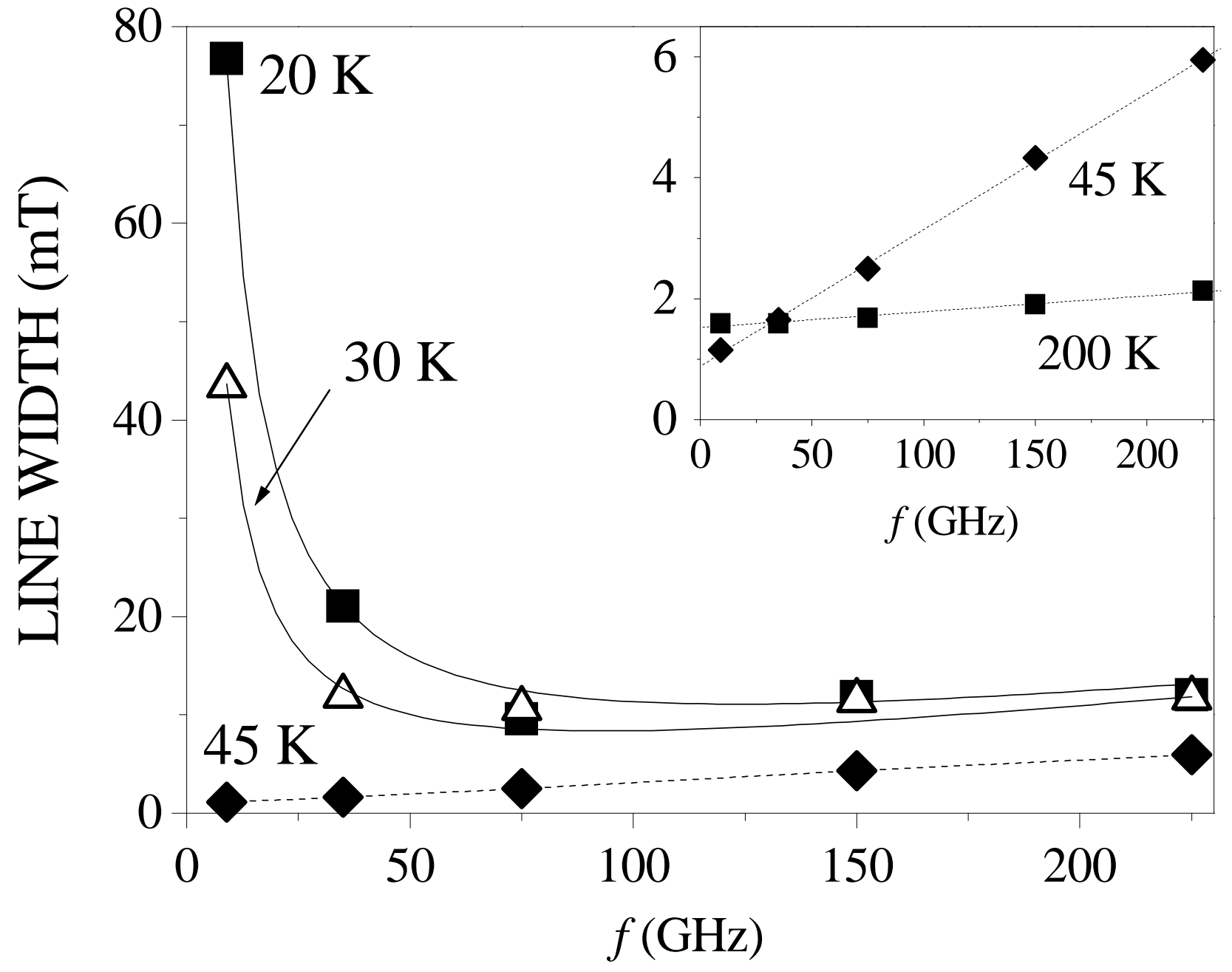




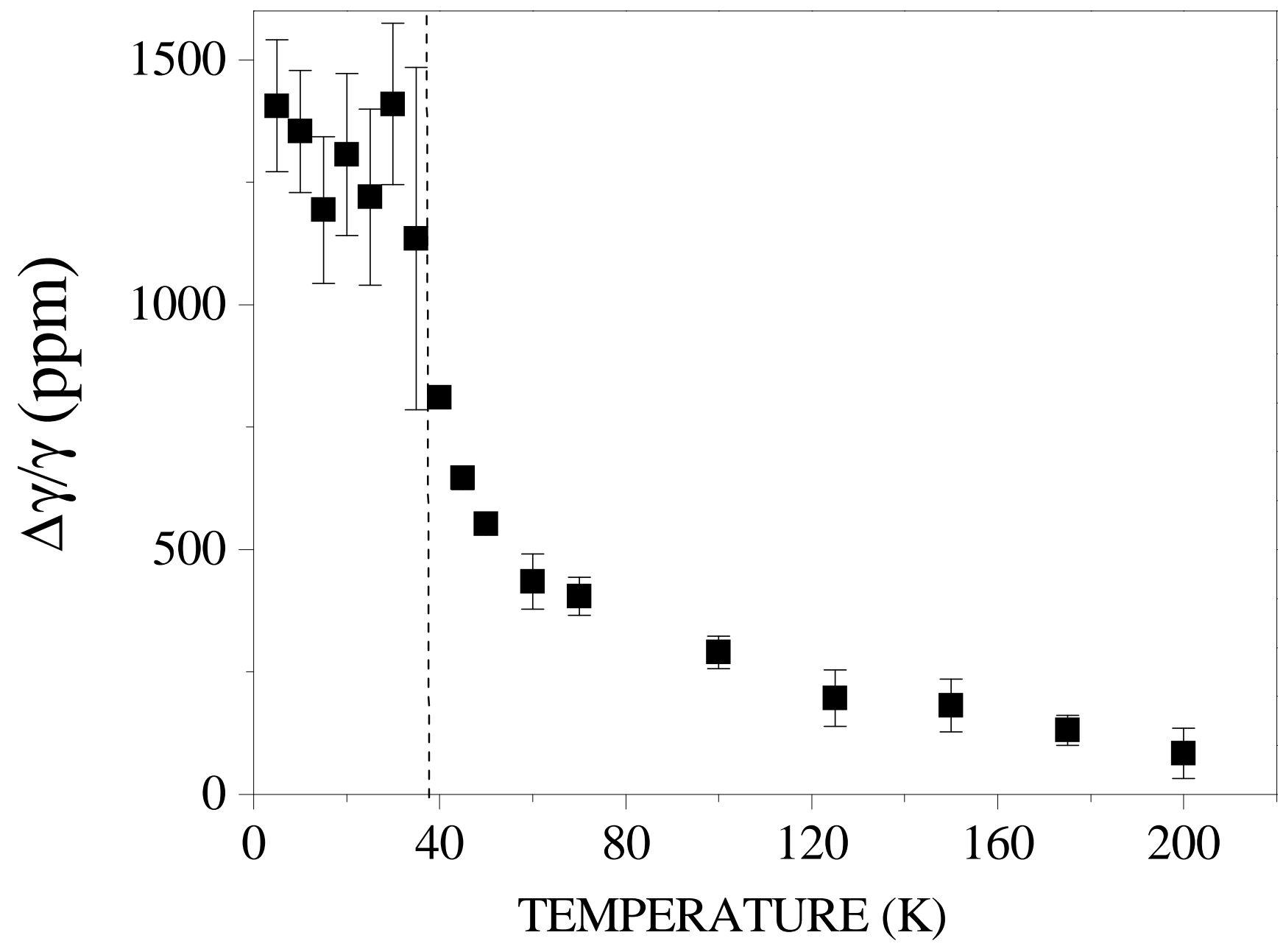

